\title{
VALIDAÇÃO DO MODELO DE ADEQUABILIDADE DE HABITAT PARA UMA GUILDA DE LAGARTOS DO BIOMA CERRADO
}

\author{
Gustavo Alves Prímola e Abreu Lima - guprimola@gmail.com \\ Universidade Católica de Brasília \\ Guilherme Crestani Vieria - guibsb01@gmail.com \\ Universidade Católica de Brasília
}

Dr. Perseu Fernando dos Santos, Ph. D - perseusan@yahoo.com.br

Universidade Católica de Brasília

Gustavo Alves Prímola e Abreu Lima - guprimola@gmail.com

Universidade Católica de Brasília 


\section{RESUMO}

$\mathrm{Na}$ história da Avaliação de Impacto Ambiental (AIA) no Brasil, dentre as metodologias que abordaram os impactos sobre a vida selvagem, existem poucos registros de métodos que adotaram a relação da fauna com seus habitats, com o objetivo de quantificar impactos. O objetivo deste estudo foi testar e validar uma metodologia de AIA, um modelo desenvolvido para o cálculo do índice de adequabilidade de habitat (IAH) para o lagarto da espécie Anolis meridionalis (Veras; Santos, 2006). O teste foi feito em três fitofisionomias de Cerrado: Campo Limpo, Campo Sujo e Cerrado Stricto Sensu, além de uma área antropizada. Todas localizadas no Jardim Botânico de Brasília. Através da amostragem populacional calculou-se a densidade de Anolis meridionalis e de espécies da guilda a qual pertencem obtendo-se um valor de Coeficiente de Correlação de Pearson, para correlacionar o Índice de Adequabilidade de Habitat com a densidade populacional, de 0,91 para o Anolis meridionalis e 0,99 para os lagartos da guilda ambos apresentando alta correlação. O modelo apresentado por Veras e Santos (2006), é aplicável quando a estimativa para o Valor de Unidades de Habitat (VUH) é feita para grandes áreas. Desta forma foi proposto uma modificação do mesmo para avaliação de Valor de Unidade de Habitat (VUH) de uma guilda de lagartos para pequenas áreas utilizando-se somente 4 variáveis do modelo anterior ou a densidade populacional específica de uma espécie ou guilda.

Palavras-chave: Anolis meridionalis, Índice de Adequabilidade de Habitat (IAH), Guilda, Parâmetros físicos do habitat, Cerrado.

\section{INTRODUÇÃO/OBJETIVO}

Grupos ou conjunto de espécies, com papéis e dimensões de nichos comparáveis dentro de uma comunidade são chamados de guildas (ODUM,1986). A guilda que abrange os lagartos da espécie Anolis meridionalis habitam diferentes fitofisionomias de cerrado.

Desta forma a guilda que abrange o lagarto Anolis meridionalis foi escolhida para avaliar as alterações geradas sobre determinado ambiente. O resultado serve de base comparativa para avaliar e quantificar perdas de habitats.

Veras e Santos (2006) dizem que:

$\mathrm{Na}$ história da AIA no país, dentre as metodologias que abordaram os impactos sobre a vida selvagem, existem poucos registros de métodos que adotaram a relação da fauna com seus habitats com o objetivo de quantificar e/ou qualificar impactos. Necessitam-se cada vez mais estudos que 
quantifiquem as perdas desses habitats como forma de avaliar os impactos ambientais sobre a vida selvagem.

Uma dessas metodologias que é conhecida como Procedimentos de Avaliação de Habitat (PAH) ou Habitat Evaluation Procedures (HEP), metodologia desenvolvida pelo US Fish and Wildlife Service (USFWS, 1980), aceita e difundida por outros órgãos norte-americanos.

Veras e Santos (2006) afirmam:

$\mathrm{O}$ PAH se baseia em princípios ecológicos e na premissa de que o habitat, para um determinado grupo de espécies selvagens, pode ser descrito como um valor numérico conhecido com Habitat Suitability Index - HSI, ou Índice de Adequabilidade do Habitat (IAH). O IAH representa, através de equação ou algoritmo, o grau de adequabilidade de um habitat para um determinado grupo de animais selvagens, da mesma espécie ou de espécies diferentes.

\section{METODOLOGIA}

O Presente estudo foi realizado no mês de outubro e novembro de 2009 em três diferentes fitofisionomias de Cerrado escolhidas. Dentre elas campo limpo, campo sujo, cerrado sensu stricto e uma área antropizada (solo compactado e vegetação atípica com ampla predominância de capim) todas elas localizadas no Jardim Botânico de Brasília JBB) (figura1). (RIBEIRO et al.,1990).

Cada fitofisionomia foi representada por uma parcela de 1ha (100x100m). Para a captura do Anolis meridionalis (figura 1) e os outros lagartos da guilda ao qual pertence, foram instaladas armadilhas de queda obedecendo a metodologia de transecto (figura $2^{\mathrm{a}}$ ) do tipo "pitfall trap" (figura 2B), que consiste de 10 armadilhas em cada parcela, totalizando 40 armadilhas, de forma a cortar diagonalmente a área de $1 \mathrm{Ha}$, formando-se uma linha reta de $141 \mathrm{~m}$ com a distribuição feita ao acaso ao longo dos dois lados do transecto.

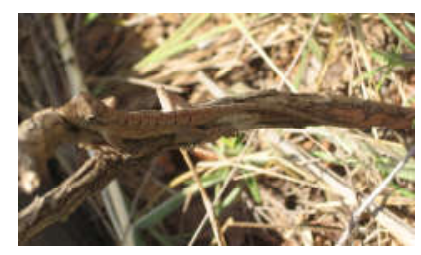

Figura 1 - Lagarto da espécie Anolis meridionalis.
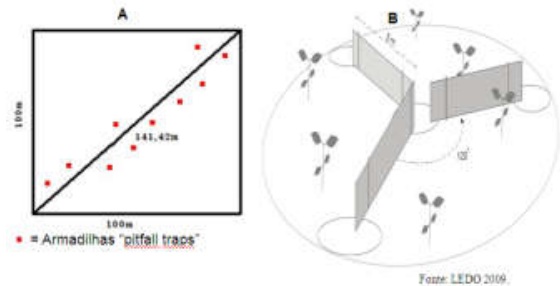

Figura 2: A - Esboço da distribuição aleatória das armadilhas. B - A armadilha de pitfall trap. 
O IAH deve ser calculado através da equação:

$I A H=[(V A V) x(V R)]^{\frac{1}{2}}$

Onde o $V_{a v}$ e o $V_{R}$ são obtidos por meio das equações (2) e (3), respectivamente.

$$
\operatorname{Vav}=\left[\left(V_{1}\right) x\left(V_{5}\right)\right]^{\frac{1}{2}}
$$

$$
\operatorname{Vr}=\left[\left(V_{2}\right) x\left(V_{3}\right) x\left(V_{4}\right)\right]^{\frac{1}{3}}
$$

Os parâmetros físicos utilizados no modelo utilizam as seguintes variáveis: (V1) \% de cobertura da camada vegetal rasteira, (V2) número de cavidades $/ 100 \mathrm{~m}^{2}$, (V3) número de cupinzeiros $/ 1000 \mathrm{~m}^{2}$, (V4) volume de madeira caída $\mathrm{em}^{3} \mathrm{~cm}^{3} / \mathrm{m}^{2}$ e (V5) \% de areia na camada superficial do solo. Sendo as variáveis V1 e V5 pertencentes ao grupo dos componentes físicos de área de vida (Vav) e as demais pertencentes ao grupo de componentes físicos de abrigo e refúgio do habitat $(\mathrm{Vr})$

A partir desses resultados encontrados, foram utilizados os gráficos de adequabilidade propostos por Veras e Santos (2006), afim de se descobrir o seu Índice de Adequabilidade.

\section{RESULTADOS E DISCUSSÃO}

Os valores obtidos pela amostragem em campo referentes às varáveis, são apresentados na tabela 1:

\begin{tabular}{|c|c|c|c|c|c|}
\hline Fitofisionomia & $\begin{array}{l}\text { Densidade da } \\
\text { camada } \\
\text { herbácea }(\%)\end{array}$ & $\begin{array}{c}\text { Número } \\
\text { de } \\
\text { buracos } \\
\text { em } 100 m^{2}\end{array}$ & $\begin{array}{c}\text { Número de } \\
\text { Cupinzeiros em } \\
1000 \mathrm{~m}^{2}\end{array}$ & $\begin{array}{c}\text { Volume de } \\
\text { madeira caída } \\
\text { no solo em } \\
\mathrm{cm}^{3} / \mathrm{m}^{2}\end{array}$ & $\begin{array}{c}\text { \% de areia na } \\
\text { camada } \\
\text { superficial do } \\
\text { solo }\end{array}$ \\
\hline Campo sujo & 72 & 9 & 5 & 283 & 94,78 \\
\hline Stricto sensu & 80 & 6 & 4 & 1784 & 93,04 \\
\hline Campo limpo & 67 & 2 & 1 & 0 & 97,01 \\
\hline $\begin{array}{l}\text { Área } \\
\text { antropizada }\end{array}$ & 92 & 2 & 2 & 0 & 96,83 \\
\hline
\end{tabular}

Tabela 1: Valores dos parâmetros físicos da amostragem de campo.

O valor de V1 obtido foi de 0,72 para o campo sujo, 0,80 para o stricto sensu, 0,67 para o campo limpo e 0,92 para a área antropizada.

Os valores obtidos de buracos e de cupinzeiros em cada fitofisionomia, foi aplicado no gráfico da variação do Índice de Adequabilidade referentes às figuras 6 e 7 
e obtido os valores referentes as variáveis 2 e 3 . Para a variável V2, os valores obtidos foram 0,6 para o campo sujo, 0,4 para o stricto sensu, de 0,15 para o campo limpo e para a área antropizada. Para a variável V3, os valores foram de 1 para campo sujo e stricto sensu, 0,2 para o campo limpo e 0,4 para a área antropizada. Aplicando-se no gráfico representado pela figura 7 , os valores encontrados na tabela 1 , obteve-se os valores de variação de IAH para o número de cupinzeiros numa área de $1000 \mathrm{~m}^{2}$. O resultado obtido para a fitofisionomia de campo limpo foi 0 para um numero contado de 1 cupinzeiro. Esse valor compromete o calculo de IAH para tal fitofisionomia, levando ao valor 0 . Pode ser explicado pelo motivo de o estudo tenha sido desenvolvido para uma área grande. Desta forma foi proposto a alteração do gráfico de forma que para o numero de 1 cupinzeiro o valor da variação de IAH não seja zero.

Os resultados do volume médio dos lançamentos foi atribuído ao gráfico da relação do Índice de Adequabilidade com o volume de madeira caída $\mathrm{em} \mathrm{cm}^{3} / \mathrm{m}^{2}$, para assim calcular o valor da variável 4. Os valores encontrados para a variável V4 foram de 0,0298 para o campo sujo e 0,0189 para o stricto sensu, já para a área de campo limpo e antropizada os valores foram zero.

As fitofisionomias de campo limpo e a área antropizada possuíam poucas árvores, o que comprometeu a amostragem feita ao acaso. Essas fitofisionomias apresentaram valores zero de madeira caída. Foi proposta a retirada dessa variável, pois levaria o cálculo do Índice de Adequabilidade de Habitat ao valor zero e apesar dessa condição, essas fitofisionomias apresentaram incidência de lagartos.

Os valores de porcentagem de areia foram usados para definição do Índice de Adequabilidade pela porcentagem de areia na camada superficial do solo e assim determinados os valores da variável 5. Os valores apresentados pela V5 são 0,85 para o campo sujo, 0,83 para o stricto sensu, 0,85 para o campo limpo e 0,86 para a área antropizada.

Com a retirada da variável V4 no cálculo do IAH (equação 1), a equação 3 foi alterada, como segue na equação 4 .

$$
V r=\left[\left(V_{2}\right) x\left(V_{3}\right)\right]^{\frac{1}{2}}
$$

Seguindo os dados das variáveis, deve-se calcular o IAH por meio das equações (1), (2) e (4).

Desta forma são obtidos os valores de IAH calculado pelas variáveis que são apresentados na coluna $\mathrm{Y}$ da tabela 2 . A coluna $\mathrm{X}$ apresenta o número de indivíduos da espécie Anolis meridionalis capturados por fitofisionomia. 
Tabela 2: Número de $A$. meridionalis capturados e o IAH obtido pelas variáveis.

\begin{tabular}{|l|c|c|c|c|}
\hline Fisionomia & $\mathbf{Y}$ & $\mathbf{X}$ & $\mathbf{X}^{\mathbf{2}}$ & $\mathbf{X} \mathbf{Y}$ \\
\hline Campo sujo & 0,7460 & 9 & 81 & 6,7140 \\
\hline $\begin{array}{l}\text { Stricto } \\
\text { sensu }\end{array}$ & 0,7178 & 5 & 25 & 3,5890 \\
\hline $\begin{array}{l}\text { Campo } \\
\text { limpo }\end{array}$ & 0,3615 & 1 & 1 & 0,3615 \\
\hline Antropizada & 0,4667 & 2 & 4 & 0,9334 \\
\hline$\sum$ & 2,2920 & 17 & 111 & 11,5979 \\
\hline
\end{tabular}

A partir desses dados, foi aplicada a equação (5) de regressão linear, usada para determinar a relação entre a variável Y e X afim de se obter o valor de IAH para o lagarto Anolis meridionalis.

$$
Y=a+b x
$$

Onde o $b$ é encontrado pela equação (6)

$$
b=\frac{\sum x y-\frac{\sum x^{*} \sum y}{n}}{\sum x^{2}-\frac{\left(\sum x\right)^{2}}{n}}
$$

O valor de a é obtido pela equação (7), considerando que o valor de $x$ é $\bar{x}$ e o valor de $y$ é $\bar{y}$.

$$
a=y-b x
$$

Com os valores de $a$ e $b$ encontrados, e aplicados na equação (5), o valor de $x$ foi considerado para cada fitofisionomia afim de se encontrar o IAH da regressão linear para cada uma.

Desta forma foram obtidos os valores de IAH para o lagarto Anolis meridionalis correlacionando as variáveis e o seu grau de incidência. Os resultados são apresentados na tabela 3.

Tabela 3: Resultado do IAH para o A. meridionalis.

\begin{tabular}{|l|c|}
\hline Fitofisionomia & IAH encontrado \\
\hline Campo sujo & 0,8006 \\
\hline Stricto sensu & 0,6089 \\
\hline Campo limpo & 0,4172 \\
\hline Antropizada & 0,4651 \\
\hline
\end{tabular}


Foi aplicado o Coeficiente de Correlação de Pearson, através da equação (8) para medir o grau da correlação entre as variáveis $\mathrm{X}$ e $\mathrm{Y}$.

$$
r=\frac{\sum\left(x_{i}-\bar{x}\right)^{*}\left(y_{i}-\bar{y}\right)}{\sqrt{\sum\left(x_{i}-\bar{x}\right)^{2 *}} \sqrt{\sum\left(y_{i}-\bar{y}\right)^{2}}}
$$

O valor encontrado para o Coeficiente de Correlação de Pearson da relação para o Anolis meridionalis foi de 0,91 .

Os valores de UH foram calculado utilizando a unidade $\mathrm{km}^{2}$ para área. Os valores da área de cada fitofisionomia foram obtidos através do estudo: Levantamento da vegetação do Jardim Botânico de Brasília - DF (RIBEIRO et al,1990).

Após a obtenção do IAH, deve-se multiplicar o valor encontrado pela área $\left(\mathrm{m}^{2}\right.$, ha, $\mathrm{km}^{2}$ ) do parque com o objetivo de se atingir o Valor da Unidade de Habitat - VUH. Apresentados na tabela 4.

Tabela 4: Valores de VUH para a onolis Meridionalis

\begin{tabular}{|l|c|}
\hline Fisionomia & Valor de VUH - Anolis meridionalis \\
\hline Campo sujo & 39085,0000 \\
\hline Stricto Sensu & 80849,0000 \\
\hline Campo limpo & 20,0000 \\
\hline Área antropizada & 0,0316 \\
\hline
\end{tabular}

Desta forma, foi proposto um cálculo de Valor de Unidade de Habitat (VUH) nas mesmas fitofisionomias para a guilda de lagartos. Para tal, foi estimado um período de captura de vinte e quatro (24) dias corridos, com captura de diversas espécies.

Para o cálculo do IAH da guilda de lagartos, foi usado os mesmos valores de Y, obtido através das variáveis usadas no cálculo do IAH para o Anolis meridionalis. Os valores de captura de todos os lagartos para cada fitofisionomia de cerrado equivalem ao valor $\mathrm{X}$ como visto na tabela 5 a seguir:

Tabela 5: Dados de IAH obtido pelas variáveis (Y) e da incidência de lagartos (X) por cada fitofisionomia.

\begin{tabular}{|l|c|c|c|c|}
\hline Fitofisionomia & $\mathbf{Y}$ & $\mathbf{X}$ & $\mathbf{X}^{\mathbf{2}}$ & $\mathbf{X}$ (Y \\
\hline Campo sujo & 0,7460 & 20 & 400 & 16,2420 \\
\hline Stricto sensu & 0,7178 & 18 & 324 & 13,9482 \\
\hline Campo limpo & 0,3615 & 2 & 4 & 0,9684 \\
\hline Antropizada & 0,4667 & 5 & 25 & 2,9500 \\
\hline$\sum$ & 2,2920 & 45 & 753 & 34,1086 \\
\hline
\end{tabular}


Foram aplicados as equações (5), (6) e (7) sobre esses valores afim de se obter o valor do Índice de Adequabilidade de Habitat para cada fitofisionomia. Os valores do Índice de adequabilidade de habitat para a guilda de lagartos estão na tabela 6 .

Tabela 6: Dados do IAH da guilda de cada fitofisionomia.

\begin{tabular}{|l|c|}
\hline Fisionomia & Valor de IAH \\
\hline Campo sujo & 0,8681 \\
\hline Stricto sensu & 0,8006 \\
\hline Campo limpo & 0,2609 \\
\hline Antropizada & 0,3621 \\
\hline
\end{tabular}

O cálculo do Coeficiente de Correlação de Pearson, equação 8 também foi aplicado para as guildas e obtido um valor de 0,99 .

Deve-se ter em conta que o Coeficiente de Correlação de Pearson, usado para medir o grau de correlação entre as variáveis físicas do seu habitat e o grau de incidência do lagarto. O valor obtido para a espécie do Anolis meridionalis foi de 0,91, o que significa que há uma alta relação entre as medições. O mesmo acontece com o Coeficiente calculado para a guilda que resultou o valor de 0,99 e assim conclui-se que possui uma maior relação dos parâmetros físicos com a incidência.

Para calcular o Valor de Unidade de Habitats (VUH), o IAH, apresentado na tabela 8 foi multiplicado pela área total de cada fitofisionomia do parque (tabela 1). Obtendose os valores de VUH da guilda, contidos na tabela 7.

Tabela 7: Valores de VUH para a Guilda

\begin{tabular}{|l|c|}
\hline Fisionomia & Valor de VUH- Guilda \\
\hline Campo sujo & 42381 \\
\hline Stricto Sensu & 106303 \\
\hline Campo limpo & 13 \\
\hline Área antropizada & 0,02 \\
\hline
\end{tabular}

A amostragem do volume de madeira caída no solo comprometeu o calculo do

IAH. E isso é explicado pelas fitofisionomias de campo limpo e antropizada possuírem poucas árvores, o que comprometeu a amostragem feita ao acaso. Essas fitofisionomias apresentaram valores zero de madeira caída. Foi proposto a retirada dessa variável, pois levaria o cálculo do Índice de Adequabilidade de Habitat ao valor zero e apesar disso essas fitofisionomias apresentaram incidência de lagartos. O que pode ser explicado pela influencia de outra fitofisionomia próxima ao local de amostragem, de maneira que o lagarto migra para essa fitofisionomia e imigra para outra, considerando-se assim a área de transição entre as vegetações. 
Os valores de Unidade de Habitat encontrados para a guilda e somente para o lagarto da espécie Anolis meridionalis apresentaram valores muito próximos, o que pode ser considerado para a aplicação do modelo também para a valoração das Unidades de Habitat da guilda. Os valores encontrados para o Anolis meridionalis e para a guilda estão na tabela 8 .

Tabela 8: Valores de VUH para a Guilda e para o Anolis meridionalis.

\begin{tabular}{|l|c|c|}
\hline Fitofisionomia & VUH - A. meridionalis & VUH - Guilda \\
\hline Campo sujo & 39085 & 42381 \\
\hline Stricto sensu & 80849 & 106303 \\
\hline Campo limpo & 20 & 13 \\
\hline Antropizada & 0,0316 & 0,02463 \\
\hline
\end{tabular}

\section{CONCLUSÕES/RECOMENDAÇÕES}

O modelo é válido para grandes áreas e propõe-se uma modificação do mesmo para avaliação de VUH para pequenas áreas utilizando-se somente de 4 variáveis, do modelo anterior (V1) \% de cobertura da camada vegetal rasteira, (V2) número de cavidades $/ 100 \mathrm{~m}^{2}$, (V3) número de cupinzeiros $/ 1000 \mathrm{~m}^{2}$ e (V5) \% de areia na camada superficial do solo ou a densidade populacional específica de uma espécie ou guilda. Desta forma, propõem-se que a equação para o cálculo de VR seja

$$
V r=\left[\left(V_{2}\right) x\left(V_{3}\right)\right]^{\frac{1}{2}}
$$

Os valores encontrados no Coeficiente de correlação de Pearson representam uma alta relação entre as variáveis e a densidade do Anolis meridionalis e as variáveis e a densidade de lagartos da guilda. Assim pode-se concluir que o modelo é válido tanto para a espécie de Anolis meridionalis como para a guilda de lagartos.

\section{REFERÊNCIAS BIBLIOGRÁFICAS}

COLLI, G.R., R. P. BASTOS, E A. F. B. ARAUJO. 2002. The character and dynamics of the Cerrado herpetofauna, p.223-231. In: The Cerrados of Brazil: Ecology and Natural History of a Neotropical Savanna. P.S. Oliveira e R. J.Marquis (eds.). Columbia University Press, New York.

COLLI, G. R.. 2005. As origens e a diversificação da herpetofauna do Cerrado. Pp. 247264 In Scariot, A., Souza-Silva, J. C., e Felfili, J. M. (eds.), Cerrado: Ecologia, Biodiversidade e Conservação. Brasília, Ministério do Meio Ambiente. 
COOPERRIDER, A. Y. Habitat Evaluation Systems. In: COOPERRIDER, A. Y.; BOYD, R. J.; STUART, H. R.Inventory and Monitoring of Wildlife Habitat. U.S. Dept. Inter., Bur. Land Manage. Service Center. Denver, CO, 1986 p. 757 - 776.

DANIELS, C.; LAMAIRE, R. Evaluating effects of water resource developments on wildlife habitat. Wildlife Soc. Bull. v.2, n.3, p. 114-118, 1974.

GIRALDELli, G. R. Estrutura de Comunidades de Lagartos ao longo de um gradiente de vegetação em uma área de Cerrado em Coxim,MS. 2007.

JUNCA, F. A.; FUNCH, L. S.; FRANCA-ROCHA, W.. Biodiversidade e Conservação da Chapada Diamantina. Brasília: Ministério do Meio Ambiente, 2005. $435 \mathrm{p}$.

LARSON, M. A.; DIJAK, W. D.; THOMPSON, F. R., III; MILlSPAUGH, J. J. Landscape-level Habitat Suitability Models For Twelve Wildlife Species In Missouri. General Technical Report NC-233. St. Paul, North Central Research Station, US Forest Service, USDA, 2003. 51p.

MACHADO, R. B., M. B. RAMOS-NETO, P.G. P. PEREIRA, E.F. CALDAS, D. A. GONÇALVES, N. S. SANTOS, K. TABOR e M. STEININGER.2004 Estimativas de perda de área do Cerrado brasileiro, p.23. Conservação Internacional, Brasília-DF.

MYERS,N.,R.A. MITTERMEIER, C.G. MITTERMEIER, G.A.B. FONSECA, E J.KENT.2000. Biodiversity hotspots for conservation priorities. Nature. 403:853-858 ODUM, Eugene P. Ecologia. Editora Guanabara. Rio de Janeiro, 1986.

PANTOJA, D. L.: Efeitos do fogo sobre a taxocenose de lagartos em áreas de Cerrado sensu stricto no Brasil Central. 2007.

RIBEIRO, J. F. ; AZEVEDO, L. G. ; SCHIAVINI, Ivan ; OLIVEIRA, P. E. A. M. . Levantamento da vegetação do Jardim Botânico de Brasília - DF. 1. ed. Brasília DF: Fundação Zoobotanica do DF, 1990. v. 1.92 p. 
ROCHA, C. C. da; FRANCA-ROCHA, W.; CHAVES, J. M.. Caracterização de unidades de paisagem utilizando avaliação ecológica rápida como suporte ao mapeamento da biodiversidade da chapada diamantina - BA. Geo UERJ - Revista do Departamento de Geografia, v. semestral, n.1, jan. 1997.

SAFRIEL, U. N.; BEN-ELIAHU, M. N. The influence of habitat structure and environment stability on the species diversity of polychaetes in vermetid reefs. In: BELL, S. S.; MCCOY, E. D.; MUSHINSKY, H. R. Habitat Structure: the physical arrangement of objects in space. St. Edmunds, Chapman \& Hall, 1991, 345-369.

SILVA, J. M. C., E J. M. BATES. 2002. Biogreographic patterns and conservation in the South American Cerrado: a tropical savanna hotspot. BioScience. 52:225233.

SCHNEIDER, M.; ALHO, C. J. R. Perdas de habitats e estado de conservação da fauna de mamíferos da bacia hidrográfica do rio Manso, MT. In: SANTOS, José Eduardo dos; CAVAlHEIRO, F.; PIRES; J. S. R.; OLIVEIRA, C. H.; PIRES, A. M. Z. C. Rodrigues. Faces da Polissemia da Paisagem - Ecologia, Planejamento e Percepção. São Carlos, RIMA, 2004. 408p.

TURNER, M. G.; GARDNER, R. H.; O’NEILL, R. V. Landscape Ecology in theory and practice: pattern and process. Nova Iorque, Springer-Verlag, 2001. 401p.

US DEPARTMENT OF THE INTERIOR. Ecological services manual of USFWS. Washington, $\quad$ USFWS, $1980 . \quad 1 \mathrm{v}$. Disponível em: http://www.fws.gov/policy/ESMindex.html. Acesso em: 14 julho. 2009.

US DEPARTMENT OF THE INTERIOR. Habitat Evaluation Procedures. 870 FW1. 1996. Disponível em: http://www.fws.gov/policy/870FW1.html. Acesso em: 14 de julho. 2009.

VERAS, V., SANTOS, P. F. 2006. Desenvolvimento do calculo do Valor de Unidade de Habitat para a espécie de lagarto Anolis meridionalis em ecossistemas de Cerrado. 